\title{
RECENT SOLAR X-RAY STUDIES IN THE UNITED KINGDOM
}

\author{
By K. A. Pounds \\ (Departement of Physics, University of Leicester. United Kingdom.)
}

RÉSUMÉ. - On passe en revue les observations de rayons $X$ solaires obtenus, ces dernières années, en Grande-Bretagne et qui comportent des événements enregistrés par le satellite Ariel, des mesures des rayons $X$ solaires de faibles énergies obtenues par le satellite NRL 1963-21 C. On donne aussi des indications sur des vols récents de fusées. On discute brièvement la nature des rayons $X$ émis par les éruptions solaires et leur mesure.

Aвsтract. - Solar X-ray data obtained over the past year by scientists in the United Kingdom are reviewed. These include several new $X$-ray events reduced from the Ariel satellite results, soft X-ray flux measurements from NRL satellite 1963-21 C and notes on recent rocket studies in this field. A brief discussion is added on the nature of solar flare $X$-rays and their measurement.

езюме. - Сделан обзор наблюдений солнечных лучей $\mathrm{X}$, полученных в эти последние годы в Великобритании и содержащих события зарегистрнрованные спутником Ариель, измения солцечных лучей X с неболышими энергиями, полученные спутником NRL 1963-21C. ириведеиы также указания о нелавних полетах ракет. Кратко обсужлена прирола лучей Х, излунаемых солнечными вспышками, и их измерение.

The present report is a continuation of the paper read at the Warsaw meeting of CosPaR (CUlHaNe et al. 1964) and reviews the new solar $\mathrm{X}$-ray data obtained in the intervening fourteen months. In section one, results are presented of several X-ray events measured by the proportional counter spectrometer on satellite Ariel I and the present position of this work is reviewed. Section two summarises the $\mathrm{X}$-ray data recorded and analysed at the Radio Research Station, Slough, from the ion chambers on board the most recent NRL satellite 1963-21C. Some notes on the present low level of non-flare solar $\mathrm{X}$-rays are given in section three, followed by a brief report on the recent successful firing of a stabilized Skylark rocket X-ray payload. In the final section is a short discussion on the measurement of solar X-rays.

\section{Ariel X-ray Spectrometer.}

$$
\begin{aligned}
& \text { (University College London } \\
& \text { and the University of Leicester.) }
\end{aligned}
$$

The design and operation of the spectrometer have been described earlier (Pounds and WILLMORE $(1963 a, b)$. Computer reduction within the past year of the direct-transmission, or highspeed, data have shown that the spectrometer operated from launch on 26 April 1962, to 1 No- vember of that year. However, detailed analysis has revealed a slight calibration shift in the spectrometer, becoming apparent after 6 May. This shift, which was due to a decrease in signal amplification, resulted in movement of the wavelength band, initially 4-14 $\AA$, toward shorter wavelengths. In principle the flight data can be corrected for this shift, but this has not been done, since, though the wavelength change involved is small, the movement toward a region of lower detector sensitivity, together with the rapid fall of incident flux to shorter wavelengths, result in generally low counting rates. The presence of a fluctuating " noise" count generated by geomagnetically trapped particles makes evaluation of such data extremely uncertain. All the results of the present section are from the period from launch to 6 May, before the occurrence of the above problem.

The McMATH working list of flares for 1962 (Dodson and Hedeman (1964)) shows a total of 24 flares and 4 subflares in the period 26 April to 5 May. X-ray records were obtained from Ariel during 12 flares and in all but one a significant enhancement in the 4-14 $\AA$ emission was observed. The exception was the importance 1 flare of 1 May commencing at 1150 U. T. No radio noise increase nor ionospheric effects have been reported either for this flare. The $\mathrm{X}$-ray record extends from 1153 to $1202 \mathrm{U}$. T., covering the reported $\mathrm{H} \propto$ maximum at $1159 \mathrm{U}$. T. The absence of 
records from sixteen flares in this period results from the satellite being in the Earth's shadow, from radiation interference and from periods of no satellite contact with a ground telemetry station.

\section{1 X-ray Profiles of some Solar Flares.}

New data of the X-ray enhancements associated with a number of flares are given in Figures 1-5. Also shown are the burst profiles of microwave solar radio noise associated with each flare. Brief notes are given below on each diagram.

An importance 1 flare was reported to commence at $1241 \mathrm{U}$. T., preceeded at 1240 by the onset of a small, but extended "gradual rise and fall" microwave burst. The X-ray counts in channels 1 and 2 refer to incident wavelengths centred on 12 and $9 \AA$ respectively and both are seen to rise significantly within minutes of the visible and radio flare onset. The increase in X-rays of $12 \AA$ becomes apparent at 1242-43 U. T., whilst that at the shorter wavelength appears to be some 4 minutes later. This measurement is discussed in section 4.

The flare noted in Figure 1 was succeeded by a second flare in the same plage region. The microwave burst shown in Figure 2 is almost certainly the sum emission from both flares. The lower half of the diagram shows the mean $12 \AA \mathrm{X}$-ray flux values for a number of satellite passes throughout this period. Points of note are the continuing X-ray enhancement at $1340 \mathrm{U}$. T., one hour after the start of the first flare, and a secondary increase between 1350 and 1400 U. T., presumably associated with the onset of the second flare. At $1520 \mathrm{U}$. T. the X-ray flux was back to its pre-flare though the microwave increase was not reported to end until $1540 \mathrm{U}$. T. This difference is probably real though the comparison of the decay of such weak enhancements, with techniques of differing sensitivities, is by no means precise.

Partial X-ray records on all five channels of the spectrometer are shown for the importance 1 flare of 1 May commencing at $1915 \mathrm{U}$. T. The respective wavelength bands are centred near 12, 9 , 7,6 and $5 \AA$. Gaps in the X-ray count record for a particular channel occur when the satellite spin points the photon counter away from the Sun during the sampling of that channel. By chance the spin period was almost precisely a sub-multiple of the spectrometer channel stepping period on 1 May ; hence the extended breaks and also continuous data runs on this day's records. The $\mathrm{X}$-ray enhancement was already large when the satellite telemetry pass began at 1917 U. T., only two minutes after the reported $\mathrm{H} \alpha$ flare onset. Thereafter the X-ray flux in each channel rose to a maximum value between
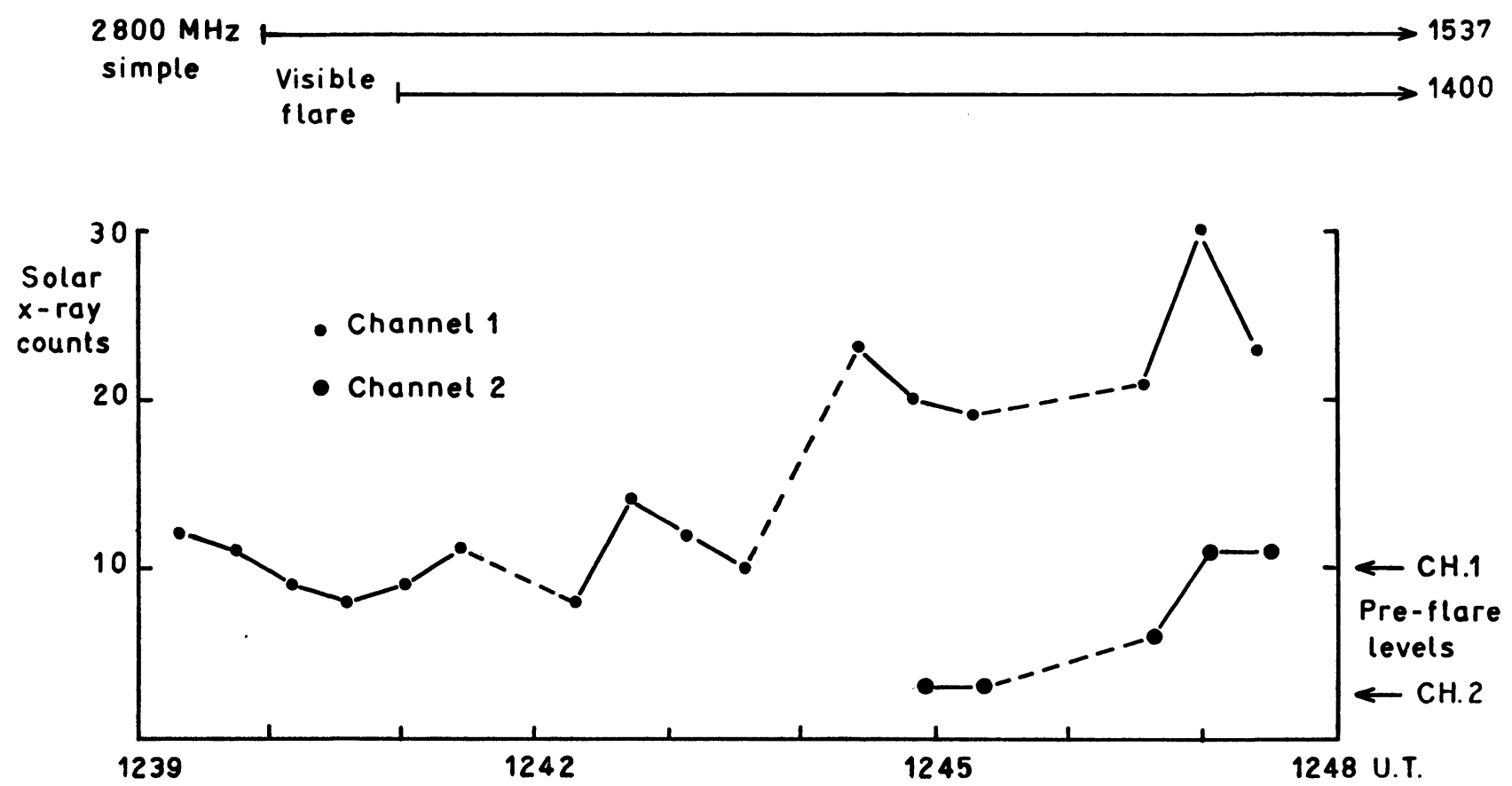

Fra. 1. - Solar flare enhancement of $12 \AA \mathrm{X}$-rays and $2800 \mathrm{MHz}$ flux.

Importance 1 flare of May 1, 1962 (1241 - 1252 - 1400 D) also importance I flare 1353.1356.1430 D). 

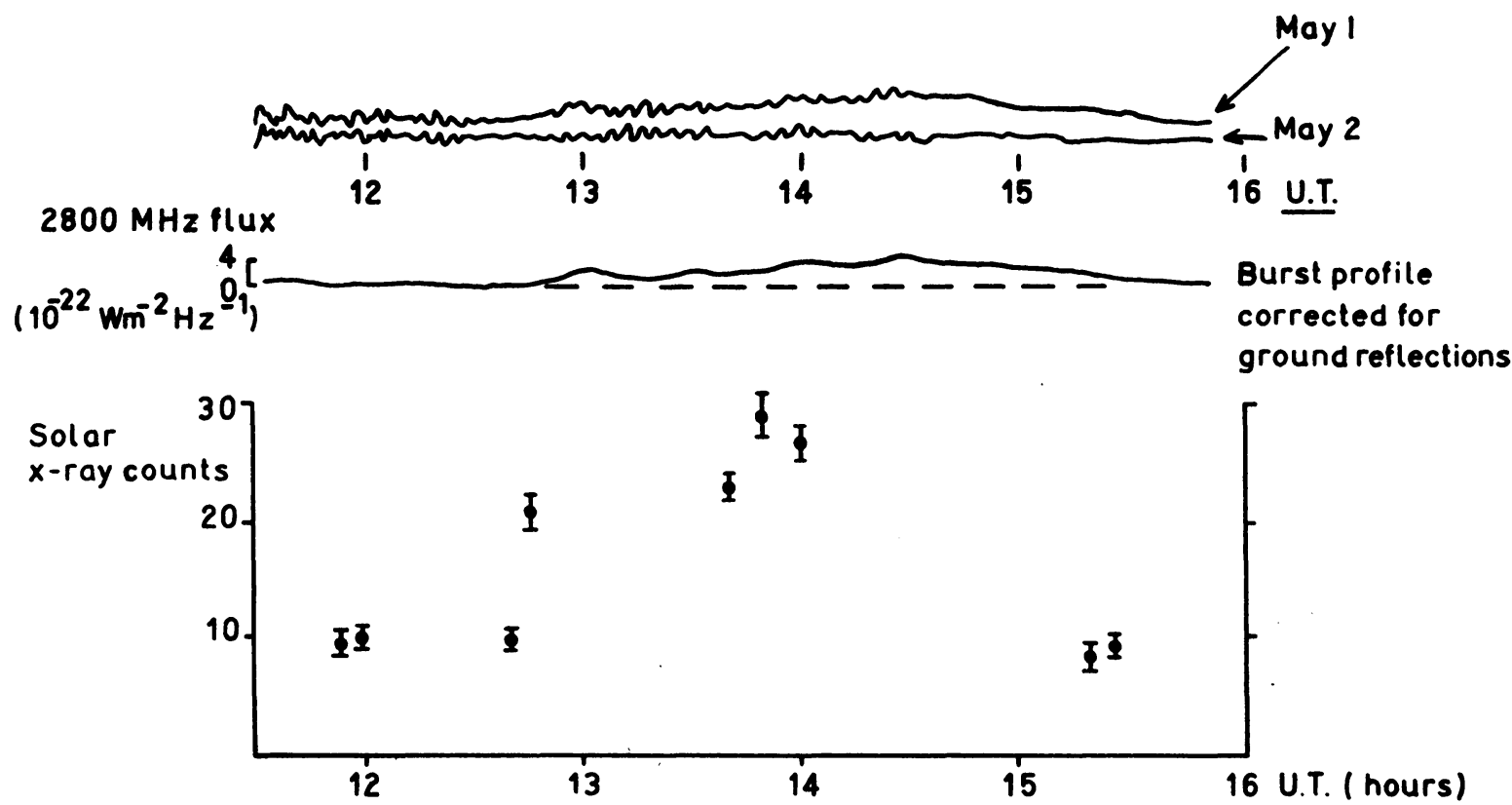

Fia. 2. - Onset of $2800 \mathrm{MHz}$ and X -ray flare enhancement, May 1, 1962.
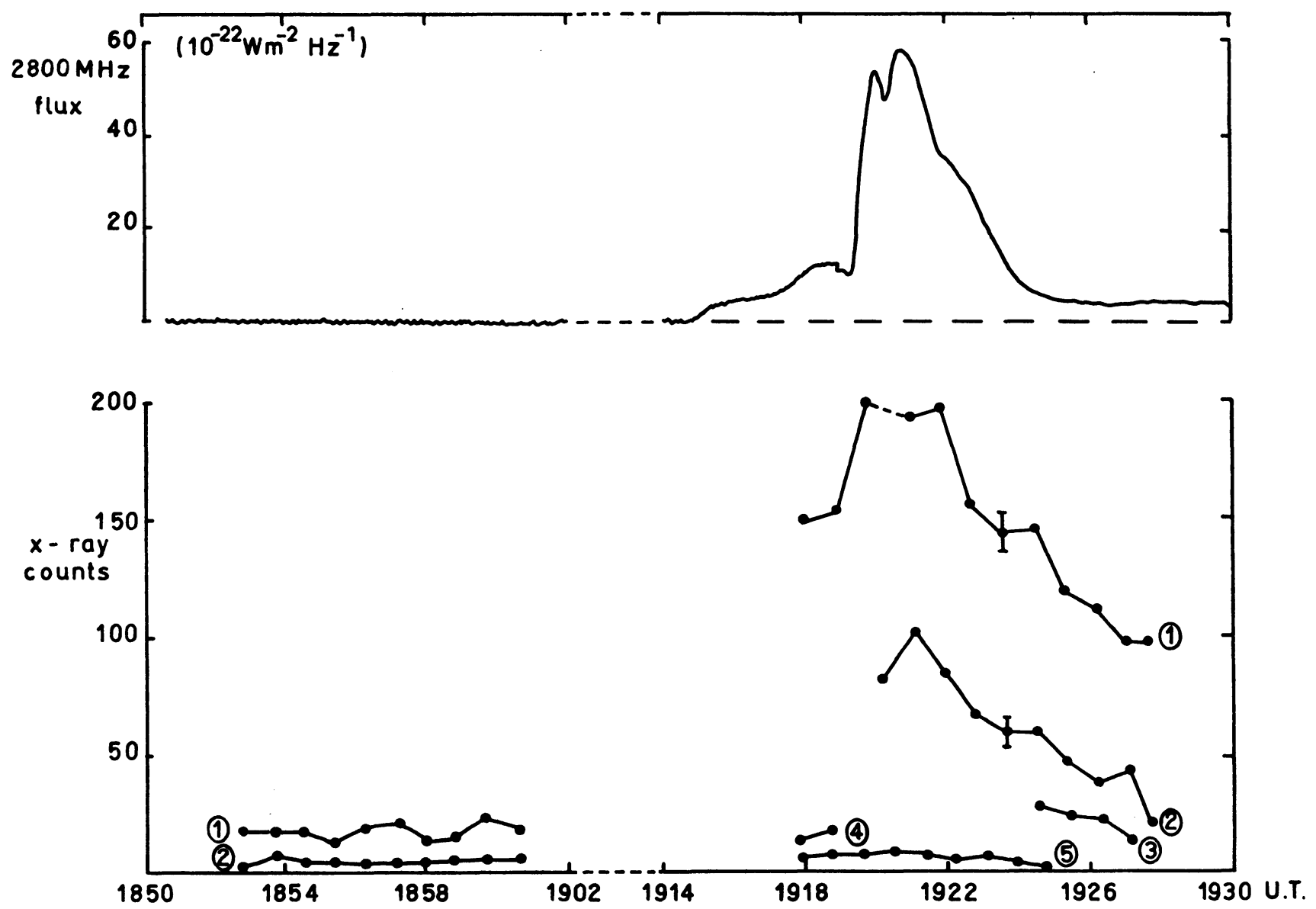

Fie. 3. - Solar flare enhancement of X-rays and $2800 \mathrm{MHz}$ flux. Importance 1 flare of May 1, 1962 (1915-1924-1940 D). 
1920 and 1921 U. T., then fell fairly rapidly to the end of the pass at $1928 \mathrm{U}$. T. Of considerable interest is the similarity between the $\mathrm{X}$-ray development and a flare-associated microwave burst. The latter began with the $\mathrm{H} \alpha$ flare at $1915 \mathrm{U}$. T rising slowly to about $1919 \mathrm{U}$. T., when a large impulsive-type burst commenced. This latter component reached a maximum at $1920 \mathrm{U}$. ' $T$. and then decayed rapidly to the pre-1919 level by 1924 U. T. Of interest is the near-coincidence of the times of peak X-ray and microwave fluxes and the appearance in both cases of an impulsive burst, superimposed on a longer lasting but lower intensity enhancement. Both radio and X-ray fluxes were considerably reduced by the time of $\mathrm{H} \alpha$ maximum at $1924 \mathrm{U}$. T. The spectral changes during the decay phase of this flare are illustrated later (Fig. 8).

The similarities between the $\mathrm{X}$-ray and microwave increases are again the most interesting features associated with this importance 2 flare. Very small flux changes over the first 24 minutes of the flare were followed by a rapid " burst" increase in both X-rays and radio noise between 1411 and 1412 U. T. No satellite data was received from 1413 to 1431 U. T., when the flux was again close to the pre-flare level. It is interesting to note that A. E. Covington at OtTAwA
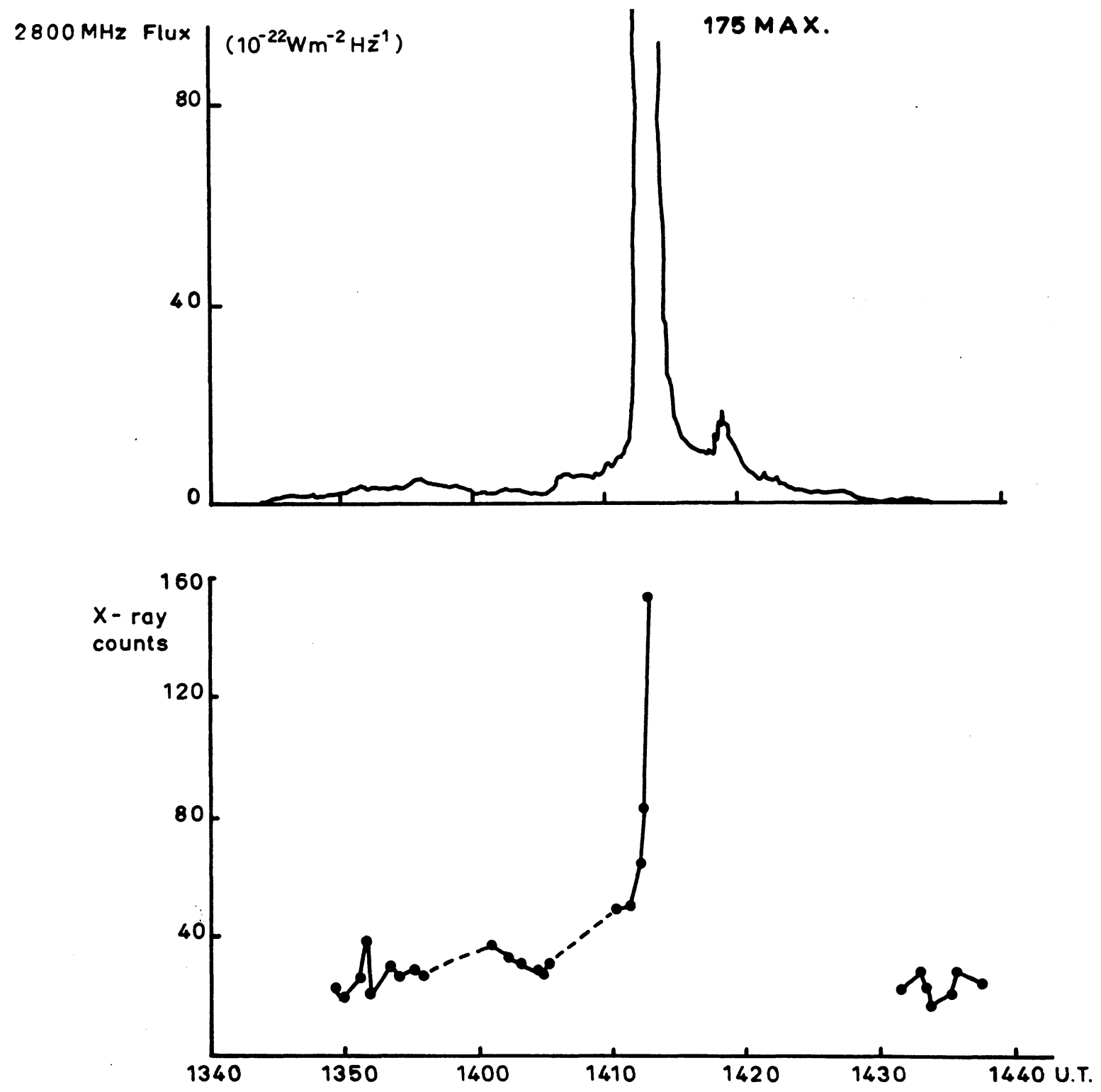

Fig. 4. - Solar flare enhancement of X-rays and $2800 \mathrm{M}_{c}$ flux imp. 2 flare of April 27, 1962 (1346-1413-1440). 
has suggested that the microwave increase almost certainly consists of an impulsive burst, from 1412-1414 U. T., superimposed on a longer lasting "gradual rise and fall" increase. These measurements are discussed in Section 3. Further points of note on this flare are the $\mathrm{H} \alpha$ maximum at 1913 U. T. and, probably more significant, the occurrence of a strong group (importance $3+$ ) of type III radio bursts between 1412 and $1416 \mathrm{U}$. T.

The spectral development of the X-ray emission from this flare is illustrated in Figure 7.

The frustrations of solar studies from a single, unstabilised satellite are demonstrated by Figures 4 and 5. In the former case the important decay phase of the X-ray burst was lost through the satellite being out of contact with a ground receiving station. With the flare shown in Figure 5, of importance 2 on 1 Mas, the satellite moved out of the Earth's shadow at 0651 U. T., just after the peak of a very large microwave burst and, as was later discovered, the occurrence of a high energy photon burst measured from satellite OSO-1. The initial increase in the $12 \AA$
X-ray flux (curve A) is due to decreasing atmospheric absorption, but the subsequent rapid fall at $0652 \mathrm{U}$. T. is of solar origin. An addition to the continuity of the $\mathrm{X}$-ray records of this flare was provided by the satellite tape recorder, showing the long enduring component of the X-ray enhancement from 0653 to beyond $0720 \mathrm{U}$. T. (It was unfortunate that during the first week launch, when most of the present data were obtained, the tape recorder was operated only intermittently.) The tape-recorded X-ray data is of lower precision, since only half the scaler stores were read on this system, but the general form of the X-ray decay of this flare is well determined.

\section{2 Flare $X$-ray Spectra.}

In general the time resolution of the spectrometer was 51 seconds, the period of two full spectral scans, to allow a " solar" and a " noise" count for all channels. To determine the incident spectral distribution with reasonable precision it was usually desirable to average the accumulated counts over several spectral scans simply on statistical grounds, but the rapid

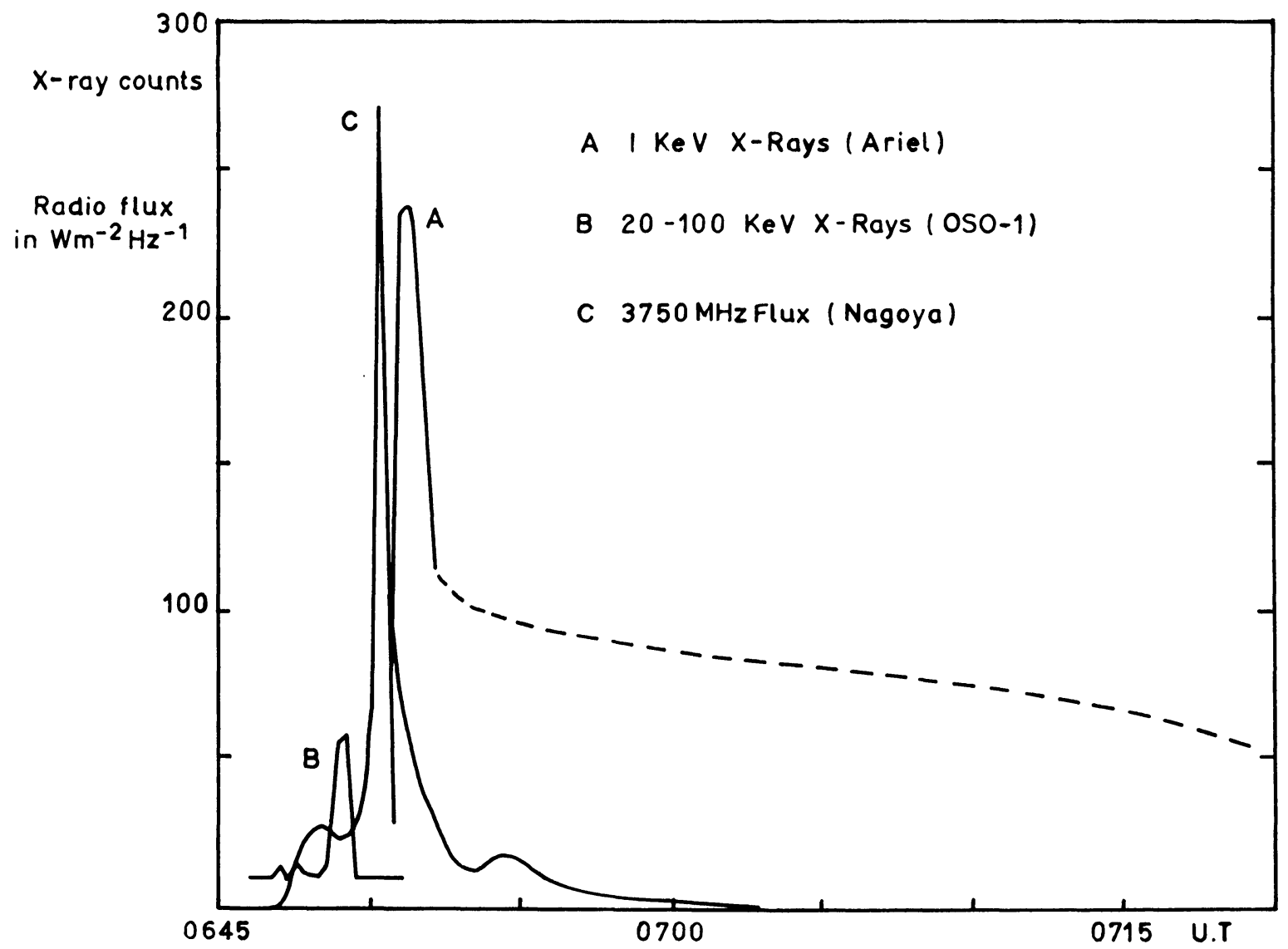

Fia. 5. - Radio and high energy photon emissions associated with imp. 2 flare of May 1, 1962 (0642-0651-0804 D). 
changes in flux occurring during flares required a compromise on this. However, despite the lack of time resolution, interesting changes in the spectral distribution of the short wavelength solar emission during a fairly representative selection of flares have been obtained. Examples are given in Figures 6-8.

This was a subflare in $\mathrm{H} \alpha$ reported to commence at 2300 U. T., on 27 April, 1962. The X-ray flux increased from $2300 \mathrm{U}$. T. and was still enhanced at $2311 \mathrm{U}$. T. when the satellite passed into the Earth's shadow. Spectra obtained during the pre-maximum period 2301-2304 U. T. and about the maximum in $\mathrm{H} \alpha$ and in $\mathrm{X}$-rays, from 2304-2307, are shown, together with a non-flare spectrum obtained two hours earlier. It is appa- rent that the flare enhancement is accompanied by a gradual " hardening" of the X-ray spectrum. In fact, if the assumption is made that the nonflare solar emission is unchanged by the occurrence of the flare, it is possible to obtain the spectra of the flare alone. These are shown (dashed) in Figure 6, and it is notable that they peak in the short wavelength band of the spectrometer.

A small complex-type microwave burst was recorded from 2300 to $2305 \mathrm{U}$. T. and a group of type III bursts, importance I, at 2300-1305 U. T.

The X-ray profile of the importance 2 flare commencing at $1346 \mathrm{U}$. T. on 27 April was shown in Figure 4. During the early part of the flare, when the X-ray enhancement was small, the spectral distribution (second and third lowest curves

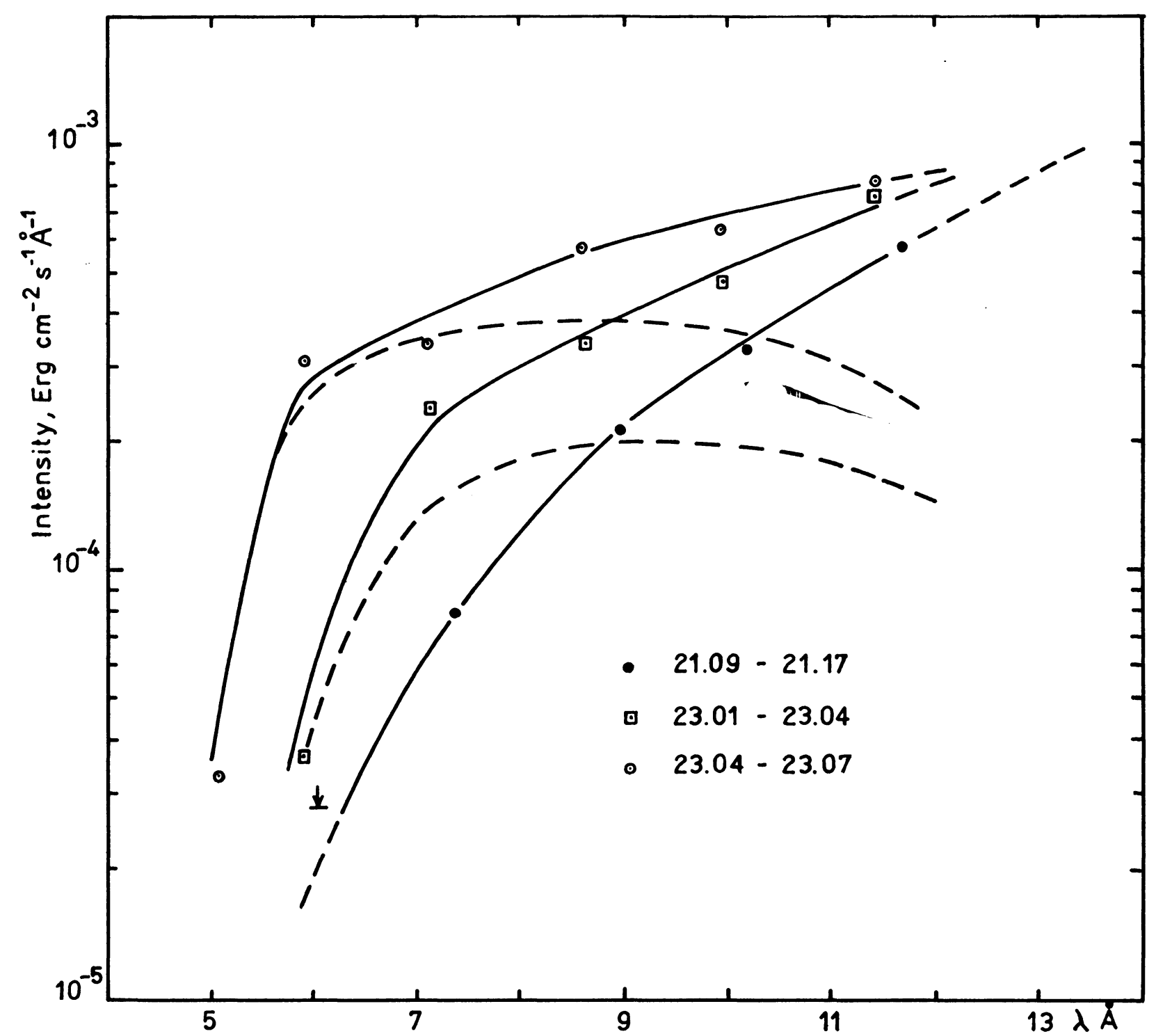

Fra. 6. - Spectral development of X-ray enhancement associated with imp. 1- flare of April 27, 1962 (2300-2305-2315). 


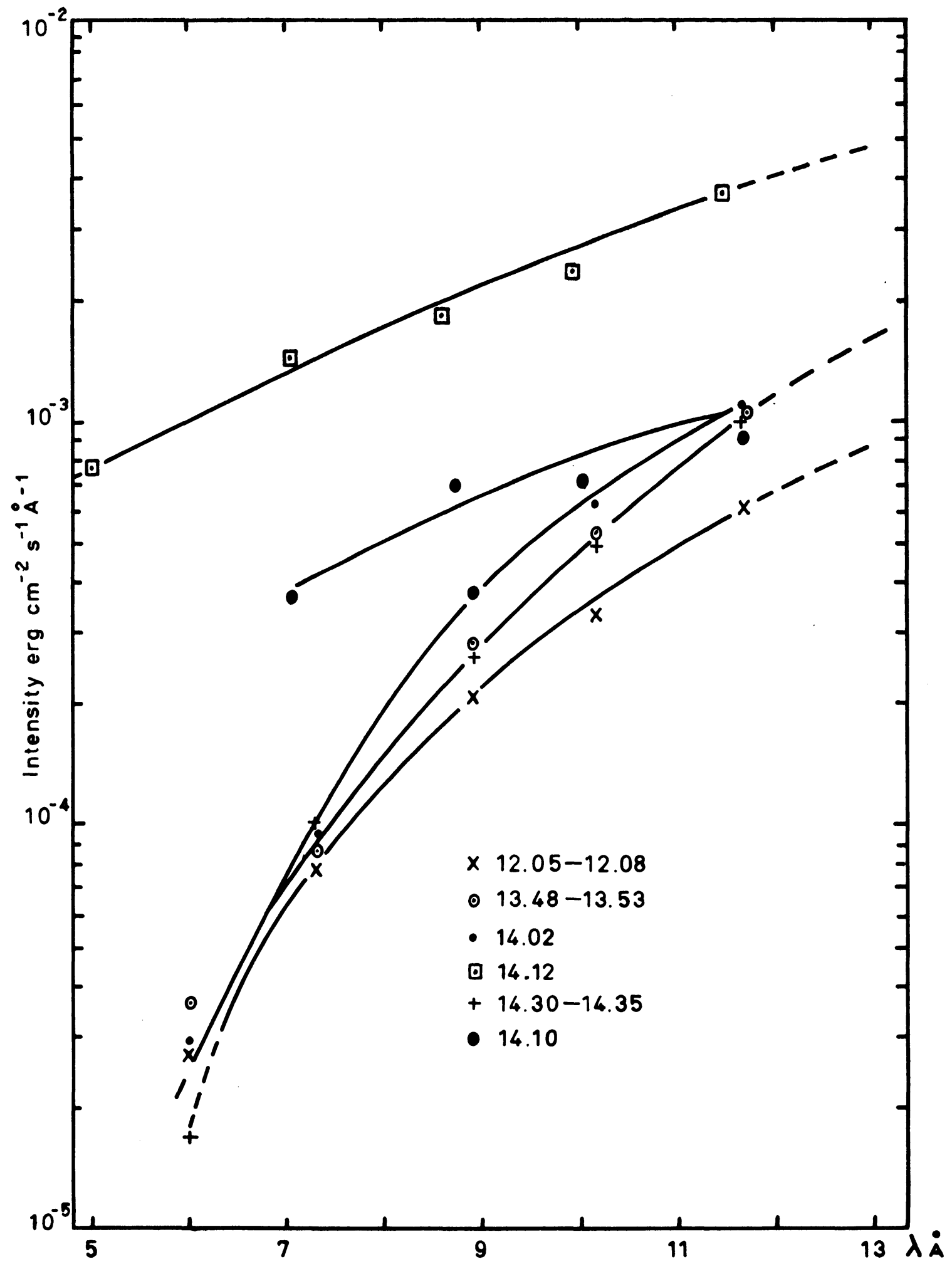

Fra. 7. - Spectral development of X-ray enhancement associated with importance 2 flare of april 27, 1962 (1346 1413-144u 


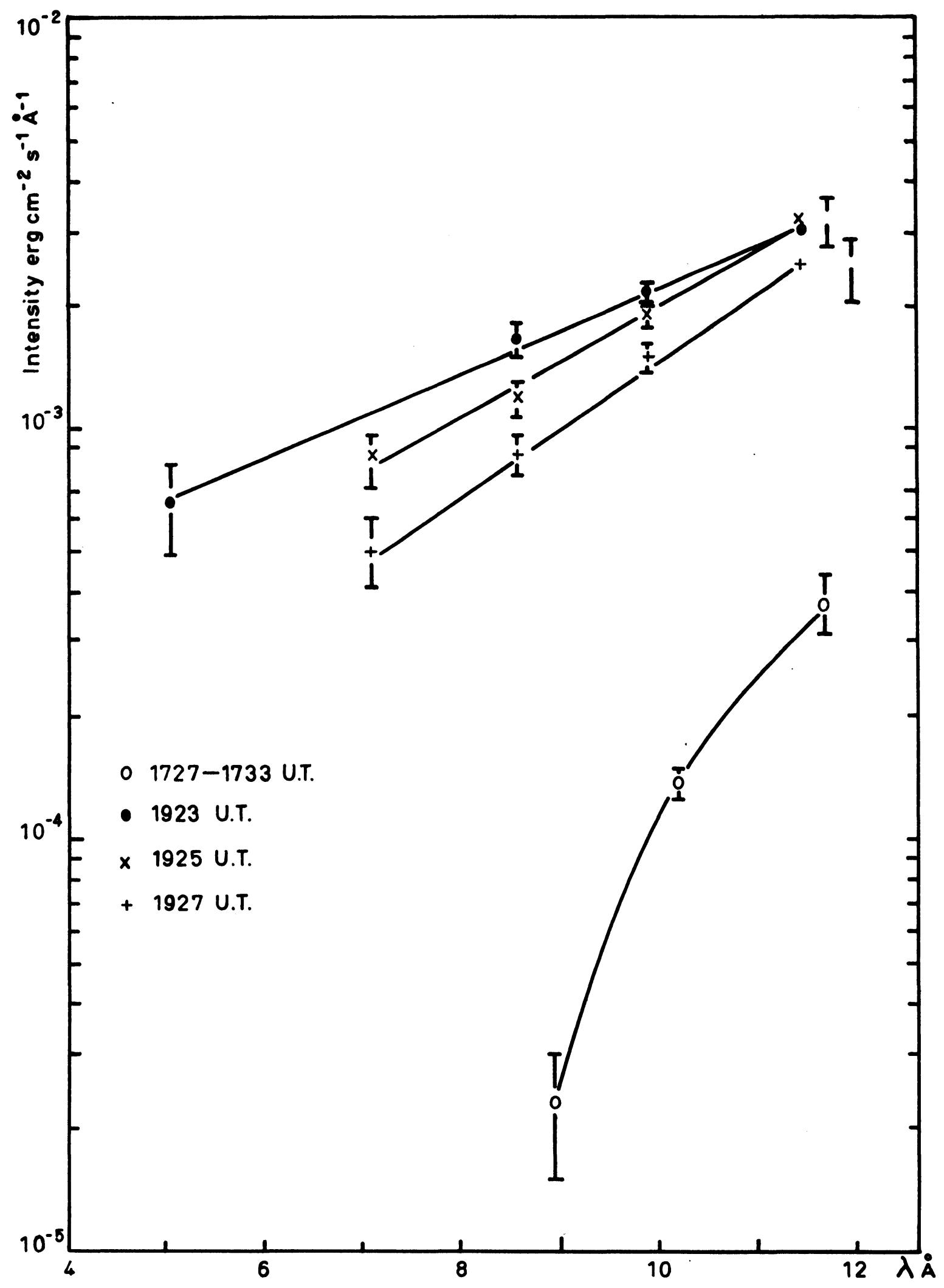

Fra. 8. - X-ray decay of imp. 1 flare 
of Figure 7) was close to that before the flare commencement (bottom curve). The spectrum at 1410 U. T., however, shows a marked hardening and at 1412 U. T., at the peak of the $\mathrm{X}$-ray burst, this harder spectrum is maintained, though with greater intensity. The similarity in spectral shape from 1410 to 1412 U. T. and the enhancement at shorter wavelength first during the " burst" phase, are notable features of this flare record. It is interesting to note that the marked ionospheric response to this flare began in the D-region (SPA starting 1410 U. T.) and moved to the E-region (SFD at 1412-1414 U. T.).

Spectra obtained during the decay phase of the flare illustrated in Figure 3 indicate a gradual " softening" of the flare X-ray spectrum. Again the flare emission is seen to dominate the sclar spectrum over the whole 4-14 $\AA$ wavelength band. The lower curve of Figure 8 was typical of the immediate pre-flare emission.

\section{1. $3 X$-rays as a Coronal Activity Index.}

Earlier reports have discussed the correlation of the level of non-flare $\mathrm{X}$-rays with coronal green line and $2800 \mathrm{MHz}$ flux and have shown that all three indices follow a general pattern determined by the density of semi-permanent active centres in the corona and chromosphere (ColHANe et al, (1964), WHITE (1964). A small number of cases have been found in the Ariel $X$-ray data where short-term increases in the $\mathrm{X}$-ray flux coincided with no reported flare. Examples are given in Figures 9 and 10. It has been pointed out before, of course, that the $X$-ray flux is likely to be a better guide to coronal activity than visible obser- vations, referring to the lower solar atmosphere. In addition, it is clear that $X$-ray flux measurements below $20 \AA$ are capable of both higher sensitivity and better time definition than visible observations.

The $12 \AA \mathrm{X}$-ray flux late on 1 May was near the mean "quiet" level for that day between 1727 and 1733 U. T. On the next satellite pass, however, this flux level was increased by 100 per cent. The importance 1 flare reported in Figures 3 and 8 was still 20 minutes in the future and no $2800 \mathrm{MHz}$ enhancement was found. The only simultaneous activity reported was an " irregular rise and fall " increase at $200 \mathrm{MHz}$ obtained a Paramaribo, listed from 1850 to $1857 \mathrm{U}$. T. with peak flux of 250 units.

The mean X-ray count per tenth second counting interval, averaged over complete passes on 2 May are shown. Each pass coincided with no reported flare or microwave activity and showed a "quiet" flux for the day of 5 to 8 counts. However, two significant enhancements were found in successive passes near 1300 U. T. and appear most likely related to the late stages of an importance 2 limb surge, commencing at 1233 U. T. and extending $2^{\prime} .8$ above the limb before $1300 \mathrm{U}$. T. If, as seems likely, the surge was associated with a flare just hidden behind the solar limb then this measurement may indicate that at least part of the $\mathrm{X}$-ray source occurred higher in the solar atmosphere than the visible flare. Comparison of a complete profile of such a surge measurement with those obtained from disc flares could be important in separating likely $\mathrm{X}$-ray mechanisms (see discussion of section 4).

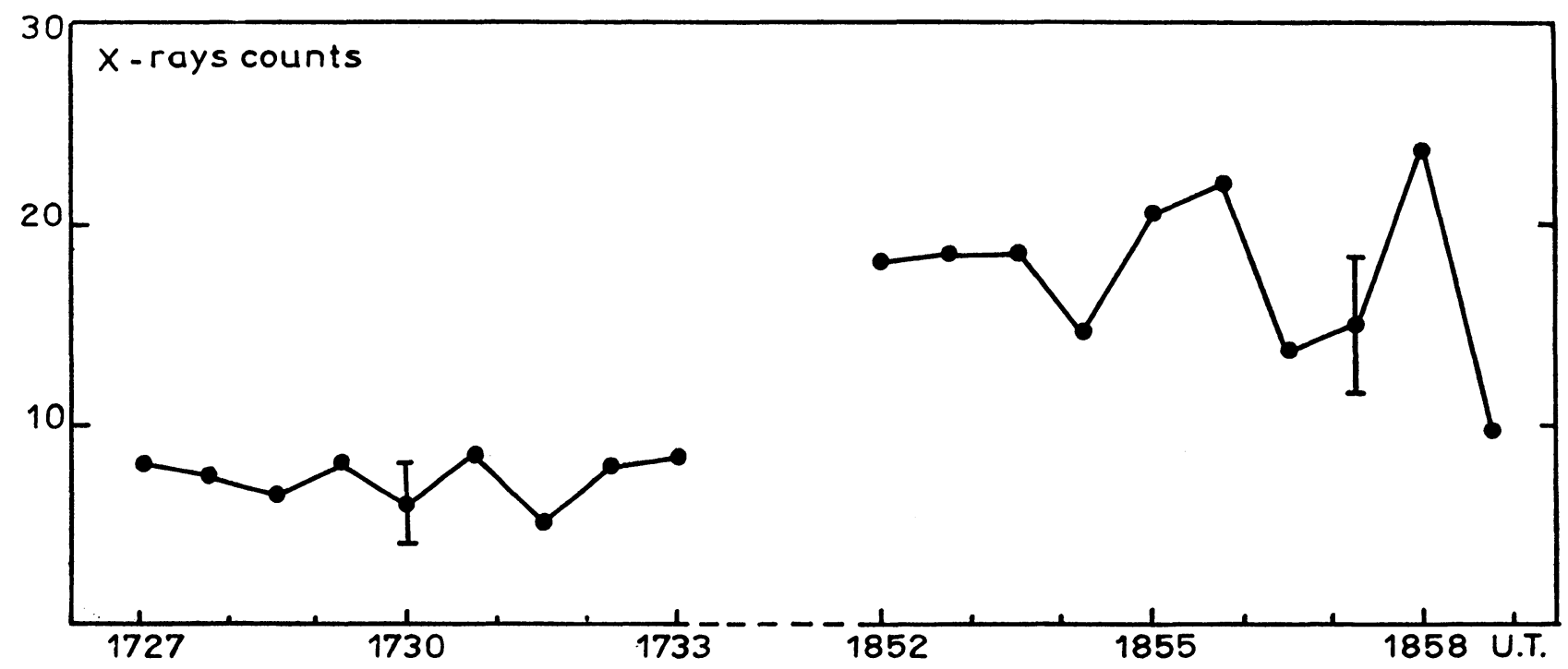

Fra. 9. - X-ray levels during 1 May, 1962. Imp. 1 flare commences 1915 U. T. 


\section{5 \\ Average $X$-ray \\ counts per \\ satellite pass}
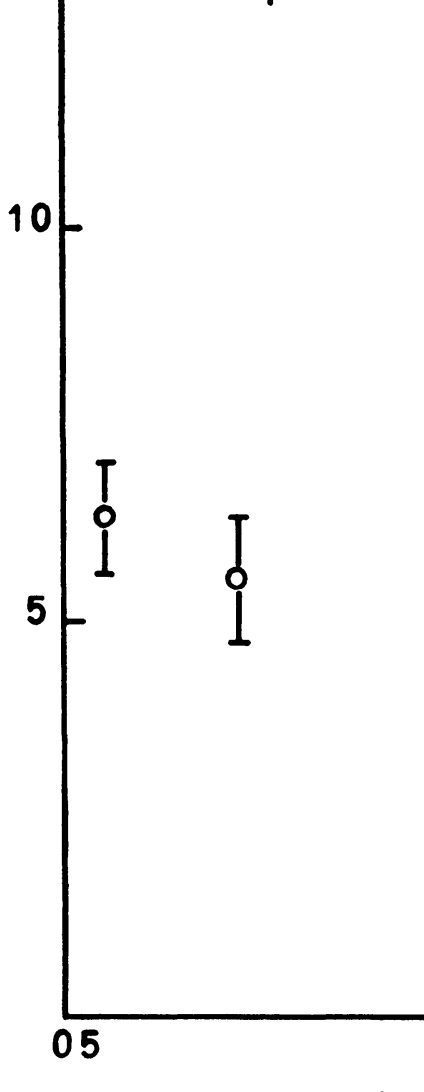

Fig. 10. - Small X-ray enhancement associated with a bright limb surge, May 2, 1962.

\section{NRL Satellite 1964-01D}

(Radio Research Station, Slough).

Monitoring the solar X-ray flux in a number of wavelength bands with an array of narrow-band ion chambers has been continued on the latest NRL piggy-back satellite 1964-01D. A team at the Radio Research Station in Slough have been recording data from this satellite since the beginning of 1964. According to the arrangement made in respect of these satellites the analysis and evaluation of such data is open to the scientists at the recording station. Figure 11 presents the complete record of mean-X-ray intensities in the 44-60 $\AA$ band recorded in each pass of the satel- lite over the Winkfield tracking station. Calculation of the absolute intensities has been made following KREPLIN (1961) by assuming an incident grey-body spectrum of $5.10^{5} \mathrm{oK}$ temperature. It is notable that throughout eight months of observation the 44-60 $\AA$ flux thus computed never fell outside the limits 1.3 to $4.5 \cdot 10^{-2} \mathrm{erg} \mathrm{cm}^{-2} \mathrm{~s}^{-2}$. The data is very largely non-flare though on a small number of occasions flares occurred during a pass, but without a noticeable effect on the X-ray flux. Comparison with the mean daily $2800 \mathrm{MHz}$ flux in Figure 11 reveals little correlation. Recordings of 8-12 $\AA$ and $<8 \AA$ fluxes are still being reduced at Slough but these do show more positive microwave ccrrelation. A fuller report of this work is being prepared for publication by $\mathrm{L}$. Thomas 

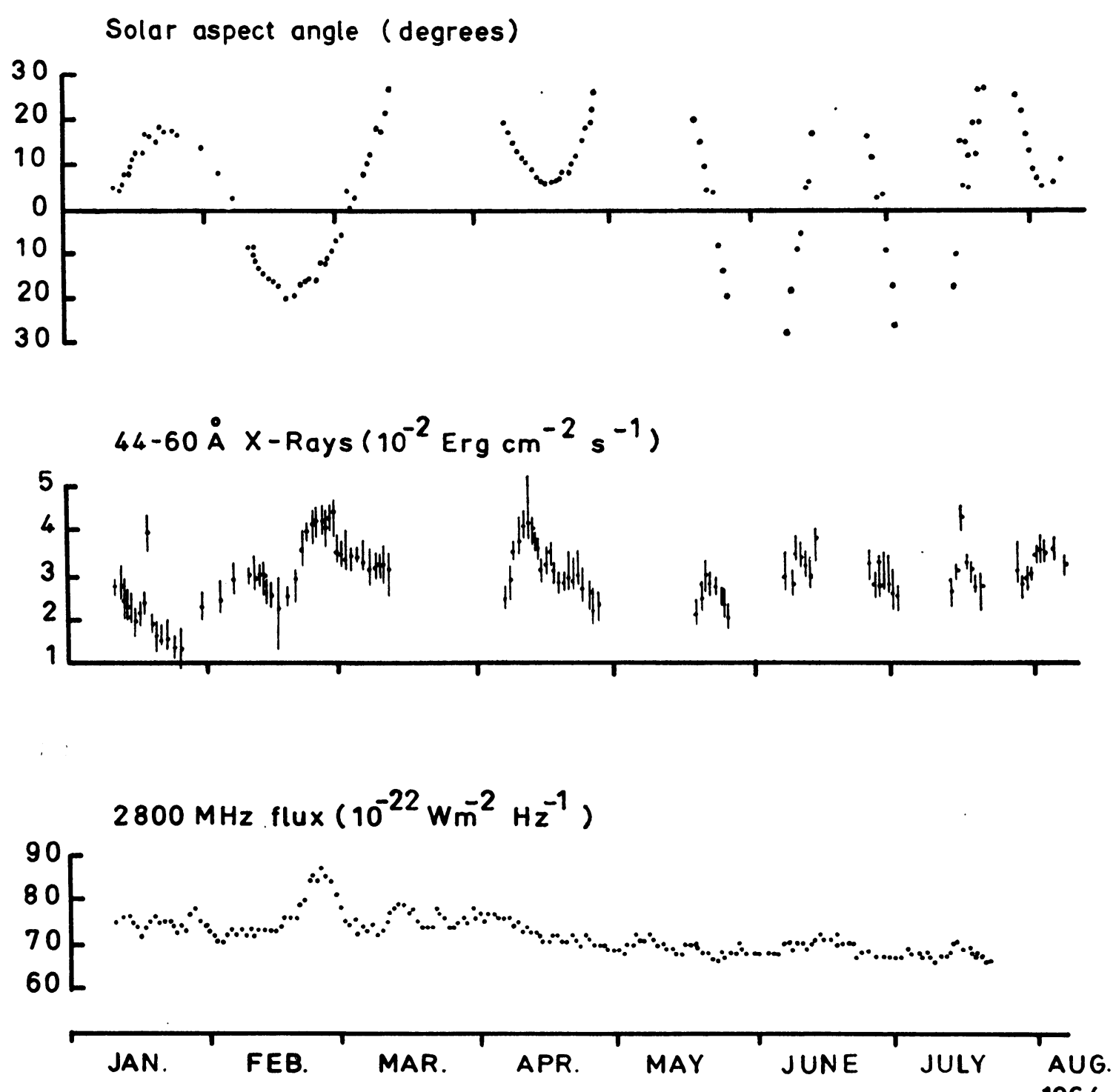

Fra. 11. - A summary of 44-60 \& measurements made through 1964 by the R. R S. Slough, England, from the N. R. L. satellite 1964-01-D.

and R. T. Venables of the Radio Research Station staff.

\section{Rocket Studies (University of Leicester).}

The use of a simple photographic device (Pounds and Bowen (1962), Pounds and SANFORD (1963)) for monitoring the $X$-ray spectrum below $5 \AA$ during Skylark rocket flights has continued over the past eighteen months. Eight satisfactory measurements have been made. Of these the full analysis of four await rocket attitude solutions, but the remainder, all occurring at very low solar activity, have given repeated measurements of what appears to be the near- minimum solar flux in this wavelength band. Combination of these measurements with those from the NRL satellite data given in section 2 provide the following figures for near-minimum X-ray fluxes :

$$
\begin{aligned}
& \left.<8 \AA=5.10^{-5} \mathrm{erg} . \mathrm{cm}^{-2} \mathrm{~s}^{-1}\right) \text { for a } 2800 \mathrm{MHz} \\
& 8-12 \AA=2.10^{-4} \text { " } \quad \text { daily index } \\
& 44-60 \AA=1 \cdot 3 \cdot 10^{-3} \quad " \quad \text { of } 74.0
\end{aligned}
$$

A valuable addition to the research facilities of solar workers in the U. K. was marked just a week ago with the flight at Woomera of a Sun stabilised Skylark rocket. A system of optical eyes and gas jets, developed by Elliott Bros, in conjunction with the Royal Aircraft Establish- 
ment, control the complete instrument section of the Skylark in yaw, pitch and roll. Preliminary reports of the firing on 11 August indicate satisfactory operation of the control system with maximum lateral deviation of 3 arc minutes and roll drift of less than 5 degrees. The scientific payload included two $\mathrm{X}$-ray experiments, prepared by Dr. P. C. RusserL and Colleagues at Leicester University, to study the solar image in several wavelength bands with an array of pinhole cameras and to obtain high resolution spectra by means of a photographic bent crystal spectrometer. Early reports indicate that the former experiment operated well and has given several $X$-ray images of the Sun in the wavelength region 10 to $60 \AA$. A report of this work will be published as soon as possible.

\section{Discussion on Solar X-RAY MEASUREMENT.}

The flare X-ray results from Ariel I, some examples of which have been presented in sections 1 and 2, show important correlation with the flare associated radio bursts, particularly at frequencies in the microwave band near $3000 \mathrm{MHz}$. These data appear to support the suggestion made in an earlier review of the Ariel results (BowEN et al, (1964)) that both thermal and non-thermal processes are involved in the $\mathrm{X}$-ray emission associated with solar flares, as is believed to be the case with the microwave bursts. Witnin the limitations of the present $X$-ray data it would appear likely that events such as those shown in Figures 3, 4 and 5 of this paper involve a dual mechanism. More generally, this may be true for the majority of flares, with the thermal process being dominant in some events and non-thermal mechanism(s) most important in others. In the writer's view there is now considerable evidence in support of non-thermal processes being important in the $\mathbf{X}$-ray production of flares, with perhaps the most likely mechanism being electron bremsstrahlung. First, there is ample optical evidence for rapid material movemenus in the "flash phase" of flares. Second, the generally accepted synchroton explanation of the impulsive microwave bursts require acceleration of electrons to several hundred kilovolts. Third, the uniform spectrum obtained during the flash phase of the $X$-ray burst shown in Figure 7 is most readily explained in terms of an increasing flux of electrons moving through a given accelerating field. Fourth, the anisotropic photon emission could provide an explanation for the old problem of the high occurrence of ionospheric crochets with flares near the limb (Dodson and Hedeman (1958)), given that the most probable electron braking would occur when the flare electrons are directed towards the dense, lower layers in the solar atmosphere.

A definitive explanation of flare $\mathrm{X}$-rays probably must await the high resolution spectral studies of individual solar regions and these should be possible in 3 or 4 years using the stabilised vehicles such as OSO and Advanced OSO. However, it is evident that considerable progress can be made with further development of proven techniques such as the counter spectrometer on Ariel I. The main requirements appear to be improved time resolution, better than 10 seconds, simultaneous cover of a wide spectral band, 0.1 to $100 \AA$, and uninterrupted measurement. Two small, stabilised satellites, equiped with tape recorders, could readily provide this data and it is interesting to note that from the aspects of frequency of spectral scanning and of absolute photometry such a technique will probably remain the best available for some years ahead.

\section{Adrnowledgments}

A large number of scientists and engineers have contributed to the work described in this report. A number appear as authors referred to in the text but acknowledgment is also due to others too numerous to mention by name. The writer would like, however, to note his particular gratitude to Mr. J. A. RATourfFe, Director of the Radio Research Station, for permission to present unpublished results from satellite 1964-01D.

Manuscrit regu le 2 novembre.

\section{REFERENCES}

[1] Bowen P. J., Norman K., Pounds K. A., SanFORd P. W. and WIILMORe A. P., 1964, Proc. Roy. Soc., 281, 538.

[2] Cullanam J. L., Willmore A. P., Pounds K. A. and SANFORD P. W., 1964, Space Research IV, North-Holland Pub. Co. Amsterdam, 741-758.

[3] Dodson H. W. and Hedeman E. R., 1958, J. Geo. Res., 63, 97. 
[4] Dodson H. W. and Hedeman E. R., 1964, IGY Solar Activity Report Series No. 25.

[5] Kreplin R. W., 1961, Ann. de Géophysique, 17, 151.

[6] Pounds K. A. and Bowen P. J., 1962, Mon. Not. R. A. S., 123, 348-357.

[7] Pounds K. A. and Sanford P. W., 1963, Proceedings of the International Conference on the Ionosphere, Inst. of Phys. and Phys. Soc., London, 513-516.
[8] Pounds K. A. and Wmimore A. P., 1963a, Space Research, III, North-Holland Pub. Co., Amsterdam, 1195-1206.

[9] Pounds K. A. and Wrilmore A. P., 1963b, Proceedings of the International Conference on the Ionosphere, Inst. of Phys. and Phys. Soc., London, 513-516.

[10] WнIте W. A., 1964, Space Research IV, NorthHolland Pub. Co. Amsterdam,

\section{Discussion}

L. Goldberg. - Have there been observations of $2800 \mathrm{MHz}$ bursts that have not been accompanied by X-ray bursts when these could have been observed?

K. A. Pounds. - In the period 26 April to 6 May there were 6 separate $2800 \mathrm{MHz}$ bursts which coincided with Ariel I X-ray records. All six showed a significant increase in $\mathrm{X}$-ray flux. In addition $\mathrm{X}$-ray increases were found for each of 3 coincident microwave bursts at $3750 \mathrm{MH}$ and on the 1 burst at $2980 \mathrm{MHz}$ On the other hand, several $\mathrm{X}$-ray increases occurred with no concurrenu microwave burst. All these X-ray enhancements were small ( $x 2$ or 3 ), however.

A. J. Deutsch. - For eventual comparison with observations that will be made of $\mathrm{X}$-ray radiation from the stars, it will be desirable to accumulate rather standardized measurements with solar flux. In the Sun and the stars, those radiations are likely toexhibit time variations that are associated with the periods of axial rotation, and with both the periods and amplitudes of the primary magnetic cycles.

R. L. F. Boyd (recorded by J. L. Stennbera). - I would like to say the same thing from the point of view of the ionospheric physicist.

Y. OHMan. - I wonder, Dr. Pounds, if the effect of isotropic radiation giving stronger intensity at the limb could have a bearing also on optical phenomena such as continuum emission in connection with flares.
I want to draw attention to a similar effect of polarization which should appear in the continuous emission spectrum when $\mathrm{H}^{-}$is formed by electron impact. I owe this information to a discussion with Dr. RuNDGREN at Pr. L. HULthen's laboratory in Sto kholm.

M. J. Seaton. - I doubt.whether one can get polarization in the $\mathrm{H}^{-}$continuum.

M. J. Seaton. - Could Dr. Pounds give a value of the temperature which he deduces from the spectral distribution of slowly decaying flare $\mathrm{X}$-rays?

K. A. Pounds. - From the study of the spectral distribution of the prolonged component of the flare enhancement, which is presumably of a thermal character, it should be possible to derive a figure for the local electron temperature. However, to do this with spectra of moderate resolution, assumptions must be made regarding the detailed distribution of energy in the continua and in the lines. Such an interpretation has been attempted for a few flare spectra using ELwERT's estimates of line continua ratios. For the flare spectra of figure 6 a figure of 10-12 million of degrees is derived. The recent work of BuRGESS showing the importance of dielectronic recombination in collision controlled plasmas must now be taken into account and, at first sight, the increase in line emission probably resulting would lead to a somewhat higher temperature for the flare $\mathrm{X}$-ray spectrum. 